\title{
Research
}

\section{Trait-dependent distributional shifts in fruiting of common British fungi}

\author{
A. C. Gange*, E. Heegaard*, L. Boddy, C. Andrew, P. Kirk, R. Halvorsen, T. W. Kuyper, C. Bässler, J. Diez, \\ J. Heilman-Clausen, K. Høiland, U. Büntgen and H. Kauserud
}

A. C. Gange (http://orcid.org/0000-0002-9918-1934) (a.gange@rhul.ac.uk), School of Biological Sciences, Univ. of London, Egham, Surrey, UK. - E. Heegaard, Norwegian Inst. of Bioeconomy Research, Fana, Norway. - L. Boddy, Cardiff School of Biosciences, Cardiff, UK. - C. Andrew, K. Høiland and H. Kauserud, Section for Genetics and Evolutionary Biology (Evogene), Dept of Biosciences, Univ. of Oslo, Blindern, Oslo, Norway. - P. Kirk, Royal Botanical Gardens, Kew, Surrey, UK. - R. Halvorsen, Geo-ecological Research Group, Natural History Museum, Univ. of Oslo, Blindern, Oslo. - T. W. Kuyper, Dept of Soil Quality, Wageningen Univ. and Research, Wageningen, the Netherlands. - C. Bässler, National Park Bavarian Forest, Grafenau, Germany. - J. Diez, Botany and Plant Sciences Dept, Univ. of California, Riverside, CA, USA. - J. Heilman-Clausen, Centre for Macroecology, Evolution and Climate, Natural History Museum of Denmark, Univ. of Copenhagen, Copenhagen, Denmark. - U. Büntgen (http://orcid.org/0000-0002-38210818), Dept of Geography, Univ. of Cambridge, Cambridge, UK, and Swiss Federal Research Inst. WSL, Birmensdorf, Switzerland, and Global Change Research Centre and Masaryk Univ. Brno, Brno, Czech Republic.

\section{Ecography}

41: 51-61, 2018

doi: $10.1111 /$ ecog. 03233

Subject Editor: Tim Newbold. Editor-in-Chief: Miguel Araújo. Accepted 11 July 2017

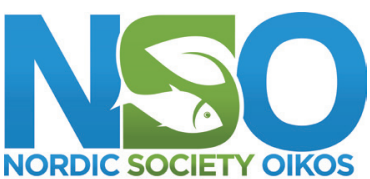

www.ecography.org
Despite the dramatic phenological responses of fungal fruiting to recent climate warming, it is unknown whether spatial distributions of fungi have changed and to what extent such changes are influenced by fungal traits, such as ectomycorrhizal (ECM) or saprotrophic lifestyles, spore characteristics, or fruit body size.

Our overall aim was to understand how climate and fungal traits determine whether and how species-specific fungal fruit body abundances have shifted across latitudes over time, using the UK national database of fruiting records. The data employed were recorded over 45 yr (1970-2014), and include 853278 records of Agaricales, Boletales and Russulales, though we focus only on the most common species (with more than 3000 records each). The georeferenced observations were analysed by a Bayesian inference as a Gaussian additive model with a specification following a joint species distribution model. We used an offset, random contributions and fixed effects to isolate different potential biases from the trait-specific interactions with latitude/climate and time. Our main aim was assessed by examination of the three-way-interaction of trait, predictor (latitude or climate) and time.

The results show a strong trait-specific shift in latitudinal abundance through time, as ECM species have become more abundant relative to saprotrophic species in the north. Along precipitation gradients, phenology was important, in that species with shorter fruiting seasons have declined markedly in abundance in oceanic regions, whereas species with longer seasons have become relatively more common overall. These changes in fruit body distributions are correlated with temperature and rainfall, which act directly on both saprotrophic and ECM fungi, and also indirectly on ECM fungi, through altered photosynthate allocation from their hosts. If these distributional changes reflect fungal activity, there will be important consequences for the responses of forest ecosystems to changing climate, through effects on primary production and nutrient cycling.

(C) 2017 Crown copyright. Ecography (C) 2017 Nordic Society Oikos

This article is published with the permission of the Controller of HMSO and the Queen's Printer for Scotland.

*Joint first author 


\section{Introduction}

Mounting evidence shows that changes in global climate have large effects on the phenology, abundance and distribution of plant and animal species (Feehan et al. 2009). With plants, earlier leafing in spring and/or later leaf senescence in autumn prolongs the growing season (Menzel and Fabian 1999, Fitter and Fitter 2002), with subsequent effects on the productivity and functioning of ecosystems, such as forests (Richardson et al. 2010).

Recent studies have shown that fungi too are highly responsive to changing climates, measured by fruit body appearance (Kauserud et al. 2012, Boddy et al. 2014, Büntgen et al. 2015). Shifts in the first and last dates of mushroom appearance have resulted in an extension of the autumnal fruiting season in the UK, and increased spring fruiting (Gange et al. 2007). These phenological changes reflect altered fungal activity, with consequences for forest ecosystem functioning, as fungi provide essential services such as decomposition, nutrient cycling and the formation of ectomycorrhizal (ECM) fungal mutualisms (Primicia et al. 2016).

Another consequence of a warming climate is a change in the spatial distribution of organisms, along altitudinal (Wilson et al. 2005) or latitudinal gradients (Hickling et al. 2006, Chen et al. 2011). However, fungi are conspicuously absent from such analyses, with just two notable exceptions. Yan et al. (2017), using species distribution modelling, predict that the distribution range in Tibet of the economically valuable Chinese caterpillar fungus Ophiocordyceps sinensis will decrease with climate warming. Meanwhile, using a global dataset of crop pest and pathogen records, Bebber et al. (2013) documented significant poleward shifts for many disease-causing fungi since 1960. Intriguingly, the northward shift by pathogens began in the late 1970s, mirroring increasing temperatures and the expansion of the overall UK fungal fruiting season (Gange et al. 2007). Given that we now know how fungal phenology responds to climate warming (Boddy et al. 2014), it is timely to assess if changes in the distribution of saprotrophic and ectomycorrhizal fungi have also occurred. To predict community responses to climate change, we need a better understanding of how species' abundances change (Johnson et al. 2013), and studying fungal distributions is critical for understanding how these organisms influence ecosystem responses to climate change (Mohan et al. 2014).

Ectomycorrhizal and saprotrophic fungi co-occur in woodlands, but differ in their nutritional mode and other life history traits (Bässler et al. 2015). Changes in spatial distributions of ECM fungi could be constrained, if there is a level of specificity between fungus and host and/or if the distribution of the host is limiting (Vellinga et al. 2009). Saprotrophs may also be constrained by host distributions (Heilmann-Clausen et al. 2016). Meanwhile, there is evidence that some ECM species can associate with novel hosts, facilitating range expansion and invasion into new habitats (Wolfe and Pringle 2012). Range expansions may lead on to fungal species becoming invasive (Kauserud et al. 2007, Gladieux et al. 2015). Furthermore, interactions between saprotrophic and ECM fungi can contribute to the structuring of fungal communities (Leake et al. 2002), with important ecosystem-level consequences (Fernandez and Kennedy 2016). Thus, understanding if changes in fungal phenology lead to changes in abundance at range edges, and ultimately shifts in distributions, is an essential, but unresolved, part of determining the fungal role in climate effects at the ecosystem level.

A fundamental goal of community ecology is to understand the distribution and abundance of organisms in time and space. Against a backdrop of climate change, the amount of information required for such analyses at the community level can be colossal, given that species show individualistic responses to climate in ecological and evolutionary time (Stewart 2009, Buckley and Kingsolver 2012). There is evidence that a trait-based approach can be of use in understanding both distribution and range shifts (Angert et al. 2011), though this has yet to be applied to fungi. Such an approach is timely, given recent syntheses of trait-based approaches in mycology (Crowther et al. 2014, Aguilar-Trigueros et al. 2015) and evidence of climate-driven reproductive trait selection in fungal communities (Andrew et al. 2016). Ultimately, a trait-based approach should enable a better understanding of processes in fungal biogeography (Martiny et al. 2006, Crowther et al. 2014). Thus, a trait-based approach provides a realistic way of testing if and how climate affects fungal abundance at the edges of ranges. Several reproductive traits related to fruit body and spore characteristics are thought to be influenced by climate variability, and may ultimately affect distributions (Kauserud et al. 2010, Andrew et al. 2016). More water is required to produce larger fruit bodies and spores, but larger spores could also be required in drier environments for germination. Dark melanised spores have better protection against UV and may, together with thick-walled spores, survive and spread further than light spores. Several other traits affect dispersal, which again can be relevant for how quickly fungi respond to climate with shifts in distributions. Larger fruit bodies produce more spores and fruit bodies with longer stipes could spread spores more distantly. In theory, smaller spores spread farther than larger ones (Norros et al. 2014) and the exterior ornamentation may also influence the aerodynamics of spores and their dispersal ability (Halbwachs and Bässler 2015).

The scarcity of studies in fungal biogeography is due in part to the fungi being invisible within substrata for long periods of time and the difficulty in characterising the huge diversity of fungi within ecosystems. While such problems become tractable using molecular methods (Tedersoo et al. 2014), these techniques cannot readily document any historical changes in fungal distributions. However, as with plant pathogens, databases of fungal occurrence can provide excellent records of presence and can be used to document changes in time and space (Bebber et al. 2013, Schenk-Jäger et al. 2016). In this study, we used a unique database of fruit body records from the UK, termed the Fungal Records Database of the British Isles (FRDBI) (<www.fieldmycology.net/FRDBI/FRDBI.asp $>$ ). The snapshot of the database was taken on 16 December 2015 and it then included 2083352 records of the fruiting of 14146 species from 
1669 to the present day, collected by amateur naturalists (citizen scientists), professional mycologists and scientific organisations.

Here, we use this database to examine whether changes in the latitudinal abundance of fruit bodies of ECM and saprotrophic fungi have occurred over the last $45 \mathrm{yr}$ in the UK. We address two specific questions: 1) has the UK latitudinal gradient in fungal abundance changed over time in a trait-specific way? 2) Are temporal shifts in fungal abundance associated with climate change? Our overall hypothesis was that changes depend on fungal traits and differ between ECM and saprotrophic species (Halbwachs et al. 2015), given that characteristics such as nutritional mode and spore size are important in determining fungal responses to climate (Damialis et al. 2015, Andrew et al. 2016). Furthermore, we hypothesized that ECM species would show fewer changes in fruiting distributions compared to saprotrophic species, because any changes in ECM fruiting will be largely dependent on changes in their host trees (Primicia et al. 2016).

\section{Methods}

\section{Data characteristics}

The data set comprised a compilation of fungal fruit body occurrence recorded throughout mainland Britain. Most records provide a collecting date and location (latitude and longitude). Many records before 1970 are deficient in some aspect (missing date or location) and we therefore used data from 1970 to 2014, which make up $84 \%$ of the full data set. From this reduced set, we extracted fruiting records of the agaricoid and boletoid members of the Agaricales, Boletales and Russulales, comprising in total 853278 records of 2844 species. Using this information, we obtained species' prevalence and recording intensities within each of the 112 Watsonian vice-counties (VC) that map the UK, for every year (Supplementary material Appendix 4 Fig. A1). We chose to use $\mathrm{VC}$ instead of gridded representation as much of the older data are at this level and this enabled us to obtain estimates of recording intensities over time. This also guided our choice of using a first order conditional autoregressive procedure rather than trying a point pattern approach with spatial Gaussian fields defined (Blangiardo and Cameletti 2015). For analyti$\mathrm{cal}$ purposes and to reduce recording bias to a minimum, we selected common (with more than 3000 records each) and widely distributed ECM and saprotrophic species that are easy to distinguish in the field (avoiding potential misidentification), with an ephemeral fleshy fruit body. Further, we eliminated species with long-lived fruit bodies or which are parasitic. The resulting data comprised 304121 records of 61 (21 ECM, 40 saprotrophic) species.

\section{Fungal traits}

Information on seven traits was taken from Knudsen and Vesterholt (2012): functional group (ECM versus saprotrophic), cap size, stipe height, spore volume, spore colour, spore wall thickness, and spore wall ornamentation. In addition, average fruiting day and standard deviation of fruiting day (a measure of season length) of each species were calculated from the data. The traits and correlations between them are explained and listed by species in Supplementary material Appendix 1 Table A1 and Fig. A1.1-A1.5.

\section{Latitude and climate}

The individual statistical unit in our analyses is the abundance of a species in a VC each year. For each VC and year, we calculated each species' average geographical position, measured by latitude and longitude. Annual mean temperature and annual total precipitation data for each record were obtained from the UK Meteorological Office, (<www.metoffice.gov.uk/climate>). These data were taken from the nine climate regions of the UK, as defined by the Meteorological Office. While we appreciate that higher resolution data may have provided more accurate representations of climate, such data were not available for the time period covered by this study. Combining the annual temperature and precipitation with the temperature difference between coldest and warmest month, we calculated a hygrothermic index (HT) reflecting the transition from continental (low values) to oceanic climate conditions (Lisewski and Ellis 2010):

$$
H T=\frac{P * T}{10 *\left(T_{H}-T_{C}\right)}
$$

where $\mathrm{P}$ is annual precipitation $(\mathrm{mm}), \mathrm{T}$ is annual mean temperature, and $T_{H}$ and $T_{C}$ are monthly mean temperature in the hottest and coldest months, respectively.

\section{Statistical analyses}

The overall goal of the statistical modelling was to quantify how species-specific abundances have changed over time across the latitudinal gradient, and to evaluate how species' traits and climate have influenced these changes. Our approach is an extension of the community assembly via trait selection (CATS) (Warton et al. 2015) following a multivariate regression specification (Jamil et al. 2013, ter Braak et al. 2017) and a joint species distribution model specification (Pollock et al. 2014, Ovaskainen et al. 2016). The key objectives were to obtain parametric estimates of how traits affected the latitudinal and climatic gradient in abundances over time, while also accounting for potential biases associated with multivariate presence-only data and uneven spatial and temporal sampling intensity.

To assess how abundance changes with time and latitude or climate, and whether changes are associated with specific traits, we compiled the number of fruit body records $\left(\mathrm{y}_{\mathrm{ijk}}\right)$ for each species i, in VC $j$ (112 VCs), and year $k$ (1970 to 2014). Because of the complex structure of the data, we used hierarchical Bayesian models (Gelman et al. 2004), fitted using the integrated nested Laplace approximation, INLA (Supplementary material Appendix 3 Table A3) (Rue et al. 2009). Because the response data $\left(\mathrm{y}_{\mathrm{ijk}}\right)$ are discrete counts and over-dispersed relative to a Poisson distribution, we assumed 
a negative binomial likelihood with a logarithmic link to a linear predictor:

$$
\begin{aligned}
& y_{i j k} \mid \theta_{i j k}, \varphi_{i j k} \sim N B\left(\mu_{i j k}, n\right) \\
& \log \left(\mu_{i j k}\right)=\eta_{i j k}
\end{aligned}
$$

Here $\theta$ denotes all parameters describing the mean, $\mu$, and $\phi$ denotes the hyper-parameters associated with parameter distributions. The parameter $\mathrm{n}$ denotes the negative binomial size, which quantifies the degree of overdispersion.

Within the linear predictor $\left(\eta_{\mathrm{i}, \mathrm{k}}\right)$, we specify parametric relationships with predictors (time, latitude, climate and traits) and several potential sources of bias common to datasets such as this, including: 1) different likelihoods of species being collected because of recorder bias; 2) yearly differences in sampling intensities; 3) spatial biases due to where observers tend to search and 4) the different sizes of VCs. These potential biases were incorporated using an offset and by a number of random effects (described in detail below) as follows, where $i=$ species, $j=\mathrm{VC}$, and $k=$ year:

$$
\begin{aligned}
\eta_{i j k}= & \log \left(q_{i} E_{j k}\right)+t_{k}+v_{j}+u_{j}+\beta_{0}+b_{0 i}+\left(\beta_{1}+b_{1 i}\right) \cdot x_{j} \\
& +\left(\beta_{2}+b_{2 i}\right) \cdot \text { time }_{k}+\left(\beta_{3}+b_{3 i}\right) \cdot x_{j} \cdot \text { time }_{k}+\beta_{4} \text { trait }_{i} \\
& +\beta_{5} x_{j} \cdot \text { trait }_{i}+\beta_{6} \text { time }_{k} \cdot \text { trait }_{i}+\beta_{7} x_{j} \cdot \text { time }_{k} \cdot \text { trait }_{i}
\end{aligned}
$$

We defined species' prevalence $\left(\mathrm{q}_{\mathrm{i}}\right)$ as the probability that a record selected at random from the full species pool belongs to species $i$. To account for variable sampling intensities both among years and between VCs, we included the sampling volume, defined as the number of records of all species for every year in each VC, $\mathrm{E}_{\mathrm{j} k}$, which is referred to as recording intensity per VC per year. This was combined with the species-specific prevalence in calculating the expected number of records per species per VC per year $\left(\mathrm{q}_{\mathrm{i}} \mathrm{E}_{\mathrm{j} k}\right)$. Using $\log \left(\mathrm{q}_{\mathrm{i}} \mathrm{E}_{\mathrm{j} \mathrm{k}}\right)$ as an offset brings the analysis into the realm of density, i.e. abundance relative to the expected number of records per species per VC per year. The logarithm was applied due to the log-link function between the linear predictor and the mean $\left(\mu_{\mathrm{ijk}}\right)$.

Subsequently, because the records were structured by year, and needed to be grouped across a VC, we included an exchangeable random contribution for the year $\left(t_{k}\right)$. Note that year as a linear term was used as part of the fixed effect in this study. Furthermore, the VCs are spatially positioned discrete units, so we included an exchangeable random contribution of the VCs $\left(\mathrm{v}_{\mathrm{j}}\right)$ and neighbouring VCs $\left(\mathrm{u}_{\mathrm{j}}\right)$. This neighbourhood structure was modelled as a Conditional Autoregressive Process (CAR) using a first-order neighbouring graph (Besag 1974), following the Besag-York-Mollie (BYM) model of spatial contribution (Blangiardo and Cameletti 2015).

To accommodate species-specific responses to the predictor variables $\left(x_{j}\right)$, we included species-specific random effects of intercept $\left(b_{0 i}\right)$, the predictor variable $\left(b_{1 i}\right)$, the time variable $\left(b_{2 i}\right)$ and the interaction between the predictor variable and the time $\left(\mathrm{b}_{3 \mathrm{i}}\right)$.

Finally, the $\beta$ 's were the fixed effects answering the main aims of the study, including how abundances are related to the effects $\left(\beta_{1}\right.$ to $\left.\beta_{3}\right)$ of time and predictors $\left(x_{j}=\right.$ latitude or climate) as well as their interactions, while $\beta_{4}$ to $\beta_{7}$ represent effects associated specifically with the investigated traits. Herein, $\beta_{7}$ is the trait-specific effect on how the gradient along the predictor changes with time, i.e. our main focus. In the study, each trait was analysed separately, and each of the predictor variables, i.e. latitude, temperature, precipitation and HT, was included separately, whereas time was always included. This produced 36 separate models, exploring all combinations of the four predictor variables with each of the nine trait variables.

In summary, the structure of the data necessitated a complex model specification with terms associated with biases and nuisance information $\left(\log (\mathrm{qE}), \beta_{0}, \beta_{1}, \beta_{2}\right.$ and $\left.\beta_{3}\right)$, with dependencies of the observations $\left(t_{k}, v_{j}\right.$ and $\left.u_{j}\right)$, with species-specific random contribution of terms $\left(b_{0 i}, b_{1 i}\right.$, $b_{2 i}$ and $\left.b_{3 i}\right)$, and finally the fixed effects of traits $\left(\beta_{4}, \beta_{5}, \beta_{6}\right.$ and $\beta_{7}$ ).

We used Gaussian weak priors for all fixed effects $(\beta$ 's), and log-gamma priors for the hyper-parameters of negative binomial size (n), the exchangeable VC contribution $\left(\mathrm{v}_{\mathrm{j}}\right)$, the spatial VC contribution $\left(\mathrm{u}_{\mathrm{j}}\right.$ ) and the temporal exchangeable contribution $\left(t_{k}\right)$. For the species-specific random contribution we were able to reduce a multivariate Wishart distribution prior to a set of univariate random contributions with individual priors of a log-gamma distribution. This reduction was possible when we used one predictor at a time and rescaled the predictor variables and the time vector to zero mean and unit variance prior to analysis. Factorial traits were analysed as a treatment contrast. All analyses were performed with the INLA package (Rue et al. 2009) in R ver. 3.3.1 (R Core Team).

\section{Data deposition}

Data available from the Dryad Digital Repository: <http:// dx.doi.org/10.5061/dryad.42k88> (Gange et al. 2017).

\section{Results}

\section{Distribution of records within the dataset}

The number of records per $\mathrm{VC}$ of the 61 focal species showed a spatial differentiation but lacked an obvious latitudinal pattern (Supplementary material Appendix 4 Fig. A1a). There was also a relatively even coverage in species richness per $\mathrm{VC}$ for these 61 species (Supplementary material Appendix 4 Fig. A1b) and almost all species were present in every VC. Meanwhile, the average abundance per species per VC over time did not show an even pattern (Supplementary material Appendix 4 Fig. A1c), with 'hotspots' of collection in some areas.

\section{Latitudinal changes in abundance}

To set the scene for assessing the trait-specific effects we illustrate the relative trend of the focal species as a group relative to the less frequent species, i.e. those included in the full species pool. This information may be inter- 
preted as bias and therefore we needed to isolate it from the information carried by the interactions involving the traits. In this we found a clear negative main effect of time $(\mathrm{p}<0.001)$, showing that recordings of the most common species have declined in average abundance over time (Fig. 1, Supplementary material Appendix 2 Table A2), and this decline was consistent across all trait groups (Supplementary material Appendix 2 Table A2). However, there was also a significant positive latitude:time interaction term (probability of being positive $=0.953$ ), indicating that the decline in abundance of common species was less at higher latitudes (Fig. 1, Supplementary material Appendix 2 Table A2).

The three-way interaction term between latitude, time, and nutritional mode was highly significant (probability of coefficient being positive $<0.001$, Fig. 1, Supplementary material Appendix 2 Table A2, Supplementary material

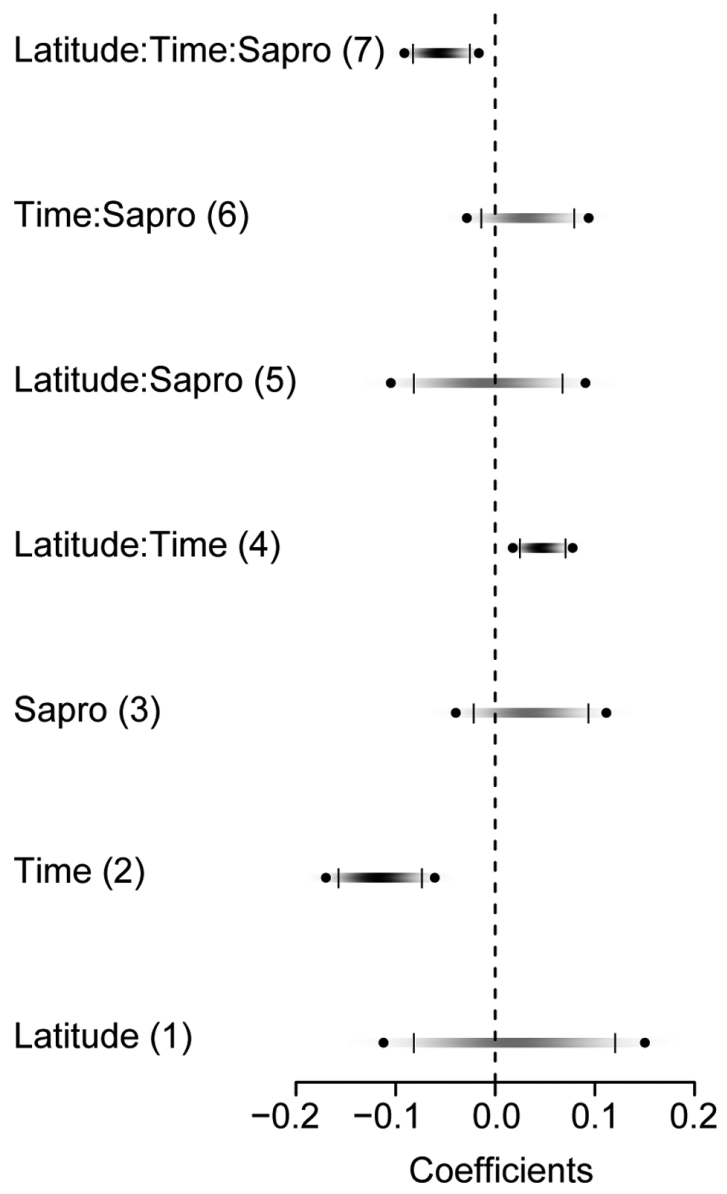

Figure 1. The posterior distribution of the coefficients for the model including the functional groups, i.e. ECM vs saprotrophic. The darkness of the individual horizontal bars reflect the probabilities (dark = high). The centre point of the horizontal bars is the expected value, and the darkest bars are those with a significant effect, i.e. the $95 \%$ credibility interval excludes 0 (for no effect). The scale of the coefficients relates to a standard deviation change of the predictor variables. Each number in brackets corresponds to those in Supplementary material Appendix 2 Table A2.
Appendix 3 Table A3), indicating that changes in abundance over time differ between ECM and saprotrophic species. As a result of this significant interaction, the modelled abundances over time and across latitudes were remarkably different for ECM (Fig. 2a) and saprotrophic species (Fig. 2b). Abundance of ECM species was relatively low in the north, but the trend has been toward increasing abundances in this area. However, in the southern region there was a marked decline in average abundance relative to the saprotrophic species over time (Fig. 2a). Meanwhile, the saprotrophic species had a more even latitudinal abundance in the early period of records than ECM species, but have declined in abundance in both the north and south, with the decline in the north more pronounced (Fig. 2b). This pattern indicates that today it is more common to record ECM species in northern parts of the UK, whereas in the south the higher average abundance of ECM records seen in the past has declined to a level similar to or lower than that of the saprotrophic species.

The probabilities associated with coefficients being positive are depicted in Fig. 3 and 95\% credibility intervals are presented in Supplementary material Appendix 2 Table A2. Three of the three-way interaction terms (Latitude:Time:Trait) are noteworthy here. Firstly, cap area showed a significant positive coefficient (Fig. 4a). The abundance of species with small caps was relatively evenly distributed from south to north at the start of the study period. The lack of a latitudinal trend persisted through time, but the abundance of small-capped fungi decreased over time. Species with larger caps, on the other hand, were previously more common in the south of the country than in the north. During the study period, this latitudinal abundance gradient shifted direction due to a strong decline in the south and a weak increase in the north (Fig. 4b).

Both spore-wall smoothness and length of the fruiting season (the standard deviation of fruiting day) have shown significant and similar latitudinal trends over time to that of functional group (Fig. 3). Fungal species with smooth spores are on average more frequently recorded today than previously, relative to species with ornamented spores (Supplementary material Appendix 2 Table A2, Fig. 4c, d). There has been a strong change among the species with ornamented spores in the south, where they have shown a considerable drop in average abundance (Fig. 4c), compared with a weak increase in the north. Species with smooth spores have declined in relative abundance in both south and north, but the decline was more pronounced in the north (Fig. 4d). Fruiting season width is another important parameter, and species with short seasons, (standard deviation of fruiting about 1 month), have become less abundant in the south, while maintaining their abundance in the north (Fig. 4e). However, species with long fruiting seasons, (standard deviation of about 2 months), have tended to become less abundant regardless of latitude, but with stronger declines in the north than in the south (Fig. 4f).

Finally, there was a weak tendency for species with thickwalled spores to be more abundant in the northern regions ('Env:trait' for 'spore wall' in Fig. 3). However, this pattern 

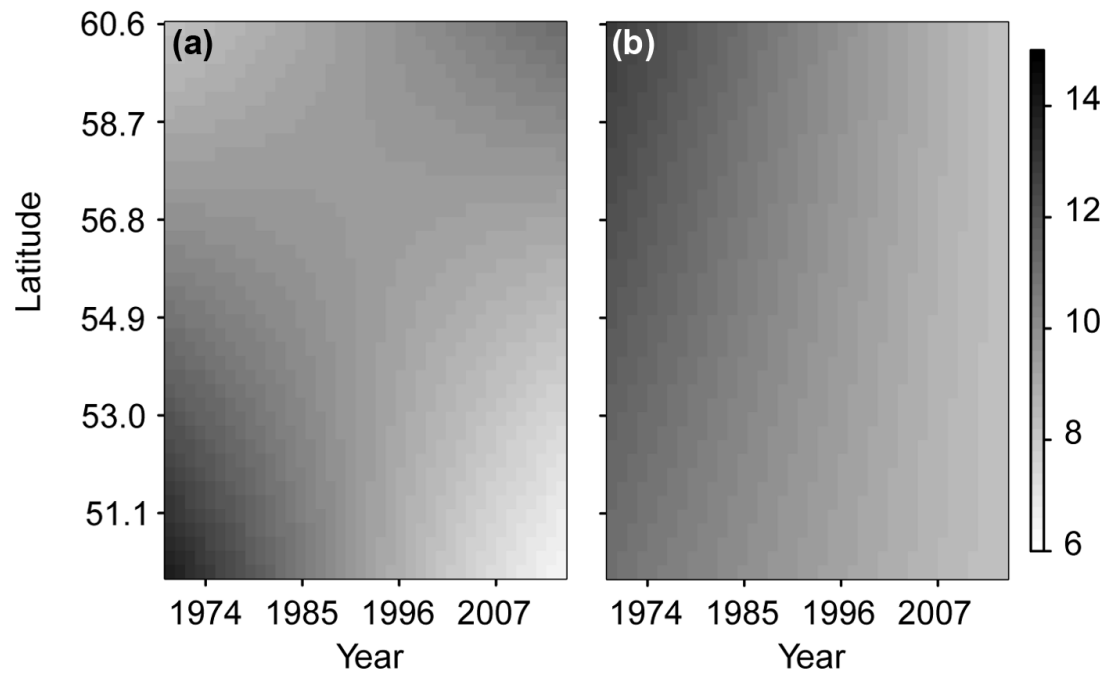

Figure 2. Diagrammatic representation of the modelled abundances over time and across latitude (northing) for hypothetical fungal species, each with a median prevalence of $\mathrm{p}=0.005$, and for a mean of 1000 records per grid cell. (a) Expectation for the functional group ECM. (b) Expectation for the functional group saprotroph. Degree of shading represents abundance, with dark colour indicating high abundance and vice versa.

did not change with time, as the three-way interaction (Latitude:Time:Trait) was not significant (Fig. 3, Supplementary material Appendix 2 Table A2).

\section{Climatic gradients}

Across the UK, temperatures decrease towards the north (Supplementary material Appendix 4 Fig. A2a), and precipitation increases toward the west (Supplementary material Appendix 4 Fig. A2b), while the hygrothermic index shows a southeast - northwest gradient (Supplementary material Appendix 4 Fig. A2c). These three climate variables each showed different, complex relationships with fungal fruiting via both direct effects and through interactions with traits and time (Fig. 3).

We found a high probability $(\mathrm{p}=0.982)$ that the threeway interaction of temperature:time:nutritional mode was positive, indicating that the influence of temperature over time differs between ECM and saprotrophs (Fig. 3, Supplementary material Appendix 2 Table A2). However, neither

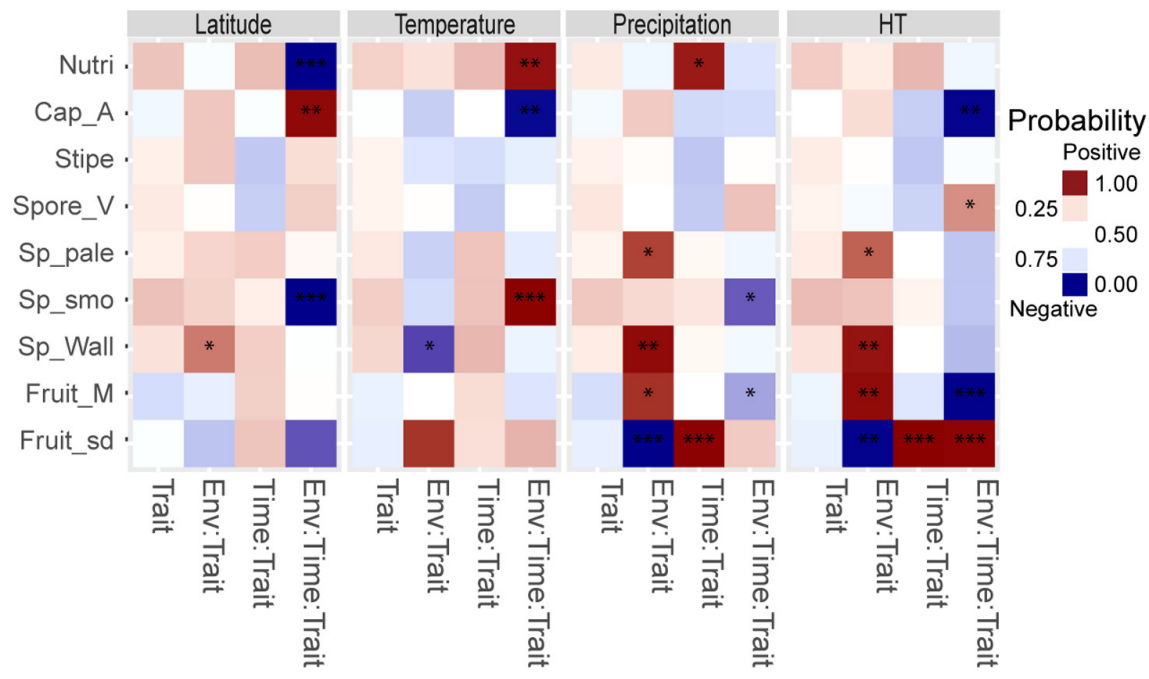

Figure 3. A colour chart depicting interaction effects of the different traits with and climate and latitude. Each trait is listed in the left of the figure. 'Env' in each legend corresponds to the particular variable: latitude, temperature, rainfall or hygrothermic index (HT). Red colours indicate a positive expected effect, whereas blue colours indicate negative effects. The intensity of the colours correspond to the probability of being either negative or positive. The stars relate to the credibility interval of the effects $* \mathrm{p}=0.9$, $* * \mathrm{p}=0.95$, and $* * * \mathrm{p}=0.99$. See also Supplementary material Appendix 2 Table A2 for the actual $95 \%$ credibility intervals associated with these effects; those depicted here are the coefficients labelled 3, 5, 6 and 7 of latitude, temperature, rainfall and hygrothermic index (HT) from Supplementary material Appendix 2 Table A2. These are the coefficients that specifically address trait-dependent distributional shifts among fruiting records in UK. 

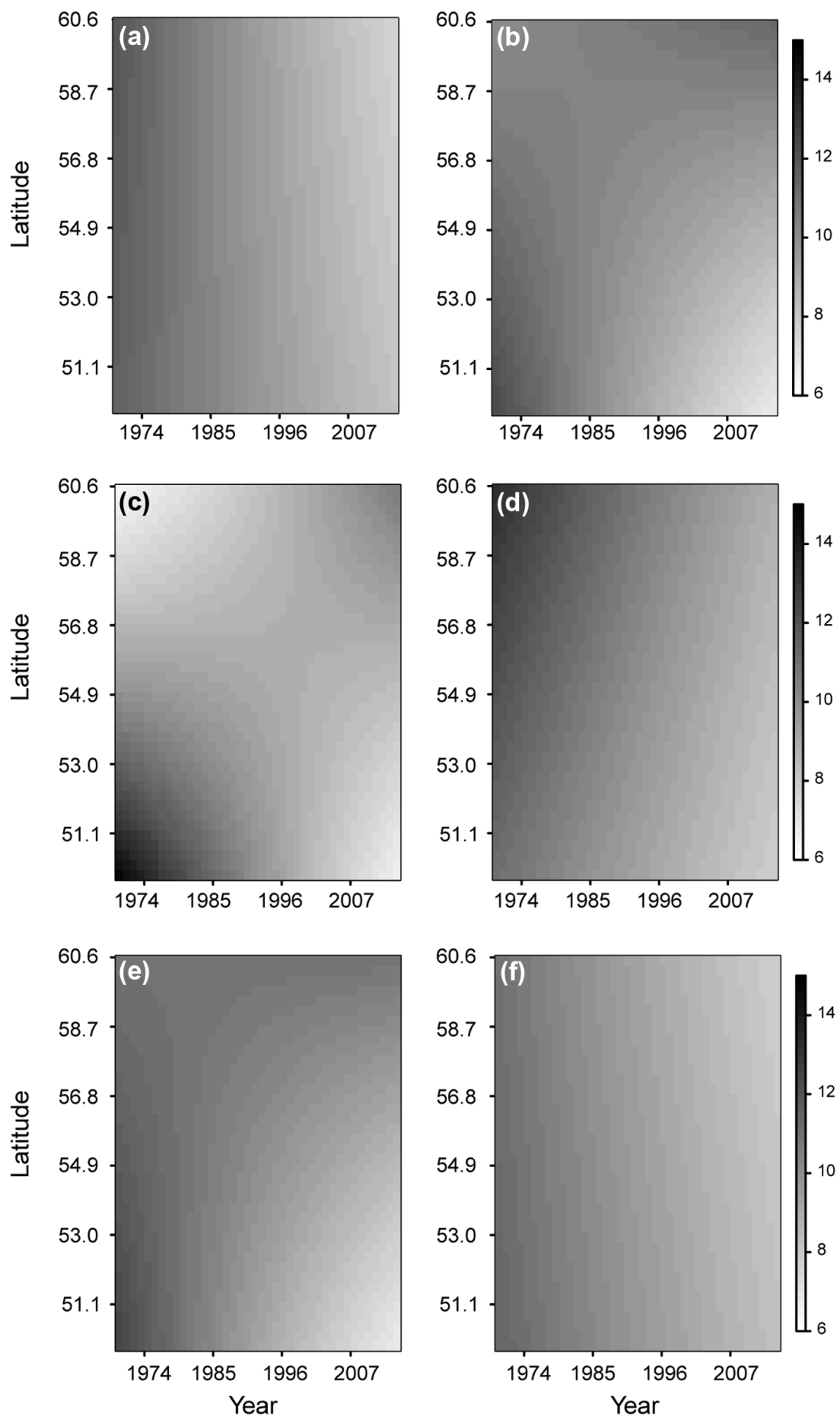

Figure 4. Diagrammatic representation of the modelled abundances over time and across latitude (northing) for hypothetical fungal species, each with a median prevalence of $\mathrm{p}=0.005$, and for a mean of 1000 records per grid cell. Expectation for species with: (a) small caps, (b) large caps, (c) ornamented spores, (d) smooth spores, (e) species with short and (f) species with long fruiting seasons. Darker shading indicates higher abundance.

rainfall nor the hygrothermic index showed a change across latitudes over time. None of the three-way interactions involving rainfall were significant, suggesting that changes in abundance across latitude over time are not related to average annual precipitation alone.
Cap area, fruiting time and length of fruiting season all showed significant three-way interactions with the hygrothermic index (Fig. 3, Supplementary material Appendix 2 Table A2), with negative coefficients for cap area and average fruiting time and a positive coefficient for fruiting 
season. Overall, we found a high probability that later fruiting species occurred more frequently in more oceanic areas (probability of coefficient being positive $=0.989$, Supplementary material Appendix 2 Table A2). The trend for species with larger caps was similar, but weaker (probability of being positive $=0.786$ ). However, over time, there has been a relative increase in abundance of later fruiters in more continental areas, relative to oceanic areas. This trend was less pronounced in the earlier fruiting species. Thus, over time, the relative difference between early and late fruiting species has reduced, leading to a high probability $(\mathrm{p}=0.998)$ that the coefficient of the three way interaction was negative (Fig. 3, Supplementary material Appendix 2 Table A2). For fruiting season length, we found a high certainty $(p=1.000)$ that the coefficient for the time:fruiting width was positive, i.e. that the temporal change in relative abundance depends on fruiting-season length. The species with broader fruiting seasons have become relatively more common compared with those of shorter fruiting-season length. The significant three-way interaction $(p=0.996$, Supplementary material Appendix 2 Table A2) suggests that this favouring of species with broader seasons has been particularly strong in regions with a high hygrothermic index (oceanic areas). However, there has been a dramatic decline in the relative abundance of the short seasoned species in the oceanic parts of UK.

\section{Discussion}

Over the last $45 \mathrm{yr}$, there has been a significant change in the distribution of fungal fruiting in the UK and this change is clearly trait-specific, upholding our original hypothesis. Among the most frequently recorded fungal species, ECM fungi now fruit more prolifically in the north of the country than in the past, but less so in the south, while saprotrophic fungi fruit less commonly now in both areas. Changes in fungal fruiting over time are strongly related to climate (seen in the hygrothermic index), suggesting that both temperature and rainfall are responsible for these distributional changes in fungal fruiting, but in association with different sets of traits.

Our most important finding is that the abundance of ECM fungal fruiting has increased in more northerly latitudes and declined markedly in the south. This finding contradicts our hypothesis that ECM fungi might show few changes over time. Autumnal mean daily temperature and rainfall in the UK have increased over our study period, but more so in the north (Anonymous 2009). Such climatic changes may result in direct effects on the ECM fungi and also in indirect effects through enhanced growth and later leaf fall of their tree hosts (Gill et al. 2015). Delayed leaf senescence in autumn has been reported across Europe (Kolarova et al. 2014), and it is perhaps no coincidence that the most prevalent host for ECM fungi in the UK, Quercus robur, has shown the greatest growing-season elongation (of 18 common tree species) of 31 d, between 1976-2010 (Kolarova et al. 2014). As the carbon supply from the host may have a strong influence on ECM fungal fruiting (Egli et al. 2010, Büntgen et al. 2012b), we conclude that temperature-driven increases in resource allocation from host trees to ECM associates, coupled with enhanced autumnal soil moisture availability, are the most likely causes of increased fruiting of ECM fungi towards the north, corroborating results from Primicia et al. (2016). The decline in ECM fungal fruiting in the south may be due more to rainfall than temperature. In the UK, over the time period covered by our study, rainfall in southern England in summer has decreased by $13 \%$, while in the north of the UK, the decrease is only 2\% (Anonymous 2009). It is known that both ECM and saprotrophic fungal fruiting are related to prevailing weather conditions between April and August (Büntgen et al. 2013). Thus, ECM fungal fruiting may have decreased in the south because of insufficient rainfall prior to fruiting. This ecological response may be comparable to the drought-induced declines of edible mycorrhizal fungal harvests in the Mediterranean area (Büntgen et al. 2012a, 2015).

Meanwhile, saprotrophic species have shown a decline in fruiting in both the north and south, with the trend being strongest in the north. Like ECM, saprotrophic fungal fruiting is also thought to be influenced by temperature and moisture effects on the process of decomposition (Rousk and Bååth 2011, Büntgen et al. 2013). Moore et al. (2008) suggest that appearance of saprotrophic fruit bodies in autumn is related to rainfall in summer. Over the study period, there has been a weak trend for fewer days of rain during the summer, a trend which is stronger in the north of the country. However, in our study the precipitation is not strongly associated with nutritional mode, i.e. the response seen among saprotrophic species is mirrored by ECM species. Thus, the latitudinal trends in changes of abundance are likely to be more driven by temperature.

Resource availability, temperature and rainfall are just three of the factors that influence the start and end of a fungal fruiting season and it is certain that these factors are inter-dependent. The hygrothermic index (combining temperature and rainfall) was a major factor related to the fruiting time and width of the fruiting season. We found that species with broader seasons have become more frequently recorded, and that this effect was stronger in more oceanic parts of the country. Meanwhile a strong decline in short-season species has occurred in the more oceanic areas. Although the fruiting season of saprotrophs in the UK is generally longer than that of ECM fungi (Kauserud et al. 2012), the patterns seen with fruiting season length did not correspond with those seen in the nutritional modes. This suggests that there is an additional climatic force acting on the fruiting season length, in saprotrophic and ECM species alike. It is likely that resource availability and a sudden drop in temperature (e.g. frost) are likely to play significant roles in determining the end of a fruiting season (Gange et al. 2013). Autumnal frost frequency has declined over the study period, but whether the timing of frosts in different parts of the country has changed is less clear (Anonymous 2009). Whether short- and broad-season species are differentially susceptible to frost is also unknown, but possible (Ohenoja 1989).

In addition to nutritional mode, other traits were important for predicting changes in latitudinal abundance. Cap 
area was a particularly important trait, as species with larger caps have become more common in the north, while those with smaller caps have declined in abundance generally. ECM fungi produce larger caps than saprotrophic fungi (Bässler et al. 2015), a phenomenon thought to be driven by the receipt of carbon from the host. The change in ECM fungal fruiting in northern areas mirrors the changes in cap area and is further evidence for enhanced resource allocation from hosts leading to increased fruit body production. However, there is also an oceanic element, not correlated with nutritional mode, as smaller cap sizes seem to be positively influenced by oceanic climate relative to species with larger cap sizes. Although, large fruit bodies need large amounts of water to expand, so also do many small fruit bodies, and the greater reduction in rainfall seen in the south may be part of the explanation of why we observed a combination of a clear nutritional mode/cap-size differentiation northwards, but also a simultaneous association with a continental gradient and cap-sizes.

A similar trend was seen with spore surface traits. Species with ornamented spores have become more abundant in the north, while smooth-walled species have become less abundant overall. ECM species often have more ornamented spore walls than saprotrophic species (Halbwachs et al. 2015) and so these changes correlate with those for nutritional mode. However, spore colour and wall thickness traits showed little change over time.

It is not just temperature and rainfall that have changed over our study period. Important changes in atmospheric $\mathrm{CO}_{2}$ levels and nitrogen deposition have occurred simultaneously and may all affect fruit body production of forest fungal species. Elevated $\mathrm{CO}_{2}$ can lead to increased fruit body production of ECM species, through enhanced carbon supply via their hosts (Andrew and Lilleskov 2009, Godbold et al. 2015). Elevated $\mathrm{CO}_{2}$ also results in altered leaf litter quality that slows decomposition (Cotrufo et al. 1994), though whether this results in reduced fruit body production of saprotrophic species is far less clear (Büntgen et al. 2013). High rates of $\mathrm{N}$ deposition reduce ECM fruit body production (Peter et al. 2001), and deposition rates have been higher in the south of the country over the study period (Fowler et al. 2004). Neither $\mathrm{CO}_{2}$ nor $\mathrm{N}$ can fully explain the increase in ECM fruiting, but both may play a role in a complex array of atmospheric factors, all of which interact (Büntgen et al. 2013).

In any analysis of this type, the biases within such datasets must be considered. Large datasets assembled by a mixture of citizen scientists and professionals can be influenced heavily by the distribution and knowledge of recorders, the geographical structure of the landscape and spatial patterns of land use (Ward 2014, Mair and Ruete 2016). However, appropriate data management and statistical methods can still yield robust analyses of changes in fungal phenology and distributions (Boddy et al. 2014, Davis et al. 2015, Taheri et al. 2016). There are several arguments for our claim that the trends found here are not caused by inherent biases in the data. Firstly, we restricted our analysis to the last $45 \mathrm{yr}$, when recording has been much more organised and coordinated, resulting in a relatively even distribution of records (Supplementary material Appendix 1-4). Secondly, because rare species can be over-represented due to collector bias (Halme et al. 2012), we analysed only a subset of common species, each with a large number of records evenly distributed across the country. Thirdly, our models explicitly account for and identify the influences of species' prevalence, sampling effort, data structure and temporal changes in recording. The latter includes changes in human population sizes, number of recorders and differences in recording intensity and recorder specialisation. Moreover, if there was recorder bias, then ECM and saprotrophic species would show similar trends, as they fruit in the same locations, but they do not. Furthermore, we identified two broad-scale distinct biological phenomena: temperature-related changes in distributions of nutritional modes and precipitation-related changes in fruiting period, which in combination make changes in recorder specialisation unlikely. As these are trait-related, it also makes bias from climatic structure unlikely (Chapman 2010). Finally, changes in forestry practices over the last $45 \mathrm{yr}$ could introduce further biases. However, in this study very few fungal species are associated with actively managed coniferous plantations, with most records from less managed pine forests, and there has been relatively little change in the latitudinal distribution of broad-leaved woodlands across the UK over our study period (Quine et al. 2011).

In summary, it is clear that distributions of fungal fruiting have changed across the UK over the last $45 \mathrm{yr}$, driven by two distinct trait specific processes acting on different climatic gradients. These changes may reflect altered resource acquisition patterns of both ECM and saprotrophic species (Bässler et al. 2016). Changes in fruiting presumably reflect changes in fungal activity, and such changes may affect ecosystem processes such as decomposition and nutrient cycling, which will have implications for the functioning and productivity of both broad-leaved forests and managed coniferous plantations (Fernandez and Kennedy 2016). Our study specifically focused on the most common species in the UK which show a decline in recording intensity relative to the less frequent species, but we do not believe that this will affect the generality of our results in the wider British mycobiota. This is because the species-specific traits show a stronger pattern than the identity of the species. For example, if a rare species has the same trait as one of the investigated common species, we can anticipate that its response will be similar to that common species, but different to an equally common one, with a different trait. Furthermore, we isolate/identify this type of bias by the main-effect of time in our models. Thus we suggest that the temperature and precipitation influences on fruiting processes are similar for the less frequent species, but this must be shown by future rigorous analyses. In addition, our results show that the exploration of historical databases to document changes in fungal fruiting abundance is a valuable approach for indicating how fungal communities may change in ecosystems such as forests, particularly in response to environmental change. 
Acknowledgements - We acknowledge all persons responsible for data collection and management.

Funding - We acknowledge the Research Council of Norway for financial support to the ClimFun project (grant 225043).

Author contributions - EH, HK, and CA designed the study, CA and PMK collated the database and resolved taxonomy, EH and JD conducted statistical analyses. ACG and EH wrote the manuscript, with substantial contributions from all authors.

\section{References}

Aguilar-Trigueros, C. A. et al. 2015. Branching out: towards a traitbased understanding of fungal ecology. - Fungal Biol. Rev. 29: 34-41.

Andrew, C. and Lilleskov, E. A. 2009. Productivity and community structure of ectomycorrhizal fungal sporocarps under increased atmospheric $\mathrm{CO}_{2}$ and $\mathrm{O}_{3}$. - Ecol. Lett. 12: 813-822.

Andrew, C. et al. 2016. Climate impacts on fungal community and trait dynamics. - Fungal Ecol. 22: 17-25.

Angert, A. L. et al. 2011. Do species' traits predict recent shifts at expanding range edges? - Ecol. Lett. 14: 677-689.

Anonymous 2009. The climate of the United Kingdom and recent trends. - DEFRA, UK.

Bässler, C. et al. 2015. Ectomycorrhizal fungi have larger fruit bodies than saprotrophic fungi. - Fungal Ecol. 17: 205-212.

Bässler, C. et al. 2016. Mean reproductive traits of fungal assemblages are correlated with resource availability. - Ecol. Evol. 6: 582-592.

Bebber, D. P. et al. 2013. Crop pests and pathogens move polewards in a warming world. - Nat. Clim. Change 3: 985-988.

Besag, J. 1974. Spatial interaction and statistical analysis of lattice systems. - J. R. Stat. Soc. B 36: 192-236.

Blangiardo, M. and Cameletti, M. (eds) 2015. Spatial and spatiotemporal Bayesian models with R - INLA. - John Wiley and Sons.

Boddy, L. et al. 2014. Climate variation effects on fungal fruiting. - Fungal Ecol. 10: 20-33.

Buckley, L. B. and Kingsolver, J. G. 2012. Functional and phylogenetic approaches to forecasting species' responses to climate change. - Annu. Rev. Ecol. Evol. Syst. 43: 205-226.

Büntgen, U. et al. 2012a. Drought-induced decline in Mediterranean truffle harvest. - Nat. Clim. Change 2: 827-829.

Büntgen, U. et al. 2012b. Linking climate variability to mushroom productivity and phenology. - Front. Ecol. Environ. 10: 14-19.

Büntgen, U. et al. 2013. Unraveling environmental drivers of a recent increase in Swiss fungi fruiting. - Global Change Biol. 19: $2785-2794$.

Büntgen, U. et al. 2015. Drought-induced changes in the phenology, productivity and diversity of Spanish fungi. - Fungal Ecol. 16: 6-18.

Chapman, D. S. 2010. Weak climatic associations among British plant distributions. - Global Ecol. Biogeogr. 19: 831-841.

Chen, I. C. et al. 2011. Rapid range shifts of species associated with high levels of climate warming. - Science 333: 1024-1026.

Cotrufo, M. F. et al. 1994. Decomposition of tree leaf litters grown under elevated CO2 - effect of litter quality. - Plant Soil 163: 121-130.

Crowther, T. W. et al. 2014. Untangling the fungal niche: the trait-based approach. - Front. Microbiol. 5: 579.

Damialis, A. et al. 2015. Fungi in a changing world: growth rates will be elevated, but spore production may decrease in future climates. - Int. J. Biometeorol. 59: 1157-1167.
Davis, C. C. et al. 2015. Herbarium records are reliable sources of phenological change driven by climate and provide novel insights into species' phenological cueing mechanisms. - Am. J. Bot. 102: 1599-1609.

Egli, S. et al. 2010. Is forest mushroom productivity driven by tree growth? Results from a thinning experiment. - Ann. For. Sci. 67: 509.

Feehan, J. et al. 2009. Climate change in Europe. 1. Impact on terrestrial ecosystems and biodiversity. A review. - Agron. Sustain. Dev. 29: 409-421.

Fernandez, C. W. and Kennedy, P. G. 2016. Revisiting the 'Gadgil effect': do interguild fungal interactions control carbon cycling in forest soils? - New Phytol. 209: 1382-1394.

Fitter, A. H. and Fitter, R. S. R. 2002. Rapid changes in flowering time in British plants. - Science 296: 1689-1691.

Fowler, D. et al. 2004. A chronology of nitrogen deposition in the UK between 1900 and 2000. - Water Air Soil Pollut. 4: 9-23.

Gange, A. C. et al. 2007. Rapid and recent changes in fungal fruiting patterns. - Science 316: 71.

Gange, A. C. et al. 2013. Mushroom phenological changes: a role for resource availability? - Proc. Natl Acad. Sci. USA 110: E333-E334.

Gange, A. C. et al. 2017. Data from: Trait-dependent distributional shifts in fruiting of common British fungi. - Dryad Digital Repository, <http://dx.doi.org/10.5061/dryad.42k88>.

Gelman, A. et al. (eds) 2004. Bayesian data analysis. - Chapman and Hall.

Gill, A. L. et al. 2015. Changes in autumn senescence in northern hemisphere deciduous trees: a meta-analysis of autumn phenology studies. - Ann. Bot. 116: 875-888.

Gladieux, P. et al. 2015. The population biology of fungal invasions. - Mol. Ecol. 24: 1969-1986.

Godbold, D. L. et al. 2015. Elevated atmospheric $\mathrm{CO}_{2}$ affects ectomycorrhizal species abundance and increases sporocarp production under field conditions. - Forests 6: 1256-1273.

Halbwachs, H. and Bässler, C. 2015. Gone with the wind - a review on basidiospores of lamellate agarics. - Mycosphere 6: 78-112.

Halbwachs, H. et al. 2015. Spore wall traits of ectomycorrhizal and saprotrophic agarics may mirror their distinct lifestyles. - Fungal Ecol. 17: 197-204.

Halme, P. et al. 2012. Monitoring fungal biodiversity - towards an integrated approach. - Fungal Ecol. 5: 750-758.

Heilmann-Clausen, J. et al. 2016. Citizen science data reveal ecological, historical and evolutionary factors shaping interactions between woody hosts and wood-inhabiting fungi. - New Phytol. 212: 1072-1082.

Hickling, R. et al. 2006. The distributions of a wide range of taxonomic groups are expanding polewards. - Global Change Biol. 12: 450-455.

Jamil, T. et al. 2013. Selecting traits that explain speciesenvironment relationships: a generalized linear mixed model approach. - J. Veg. Sci. 24: 988-1000.

Johnson, N. C. et al. 2013. Predicting community and ecosystem outcomes of mycorrhizal responses to global change. - Ecol. Lett. 16: 140-153.

Kauserud, H. et al. 2007. Asian origin and rapid global spread of the destructive dry rot fungus Serpula lacrymans. - Mol. Ecol. 16: 3350-3360.

Kauserud, H. et al. 2010. Climate change and spring-fruiting fungi. - Proc. R. Soc. B 277: 1169-1177.

Kauserud, H. et al. 2012. Warming-induced shift in European mushroom fruiting phenology. - Proc. Natl Acad. Sci. USA 109: 14488-14493. 
Knudsen, H. and Vesterholt, J. (eds) 2012. Funga Nordica, 2nd ed. - Nordsvamp.

Kolarova, E. et al. 2014. Long-term temporal changes in central European tree phenology (1946-2010) confirm the recent extension of growing seasons. - Int. J. Biometeorol. 58: 1739-1748.

Leake, J. R. et al. 2002. Interactions between ecto-mycorrhizal and saprotrophic fungi. - In: van der Heijden, M. G. A. and Sanders, I. R. (eds), Ecological studies: mycorrhizal ecology. Springer, pp. 345-372.

Lisewski, V. and Ellis, C. J. 2010. Epiphyte sensitivity to a cross-scale interaction between habitat quality and macroclimate: an opportunity for range-edge conservation. - Biodivers. Conserv. 19: 3935-3949.

Mair, L. and Ruete, A. 2016. Explaining spatial variation in the recording effort of citizen science data across multiple taxa. - PLoS One 11: e0147796.

Martiny, J. B. H. et al. 2006. Microbial biogeography: putting microorganisms on the map. - Nat. Rev. Microbiol. 4: 102-112.

Menzel, A. and Fabian, P. 1999. Growing season extended in Europe. - Nature 397: 659-659.

Mohan, J. E. et al. 2014. Mycorrhizal fungi mediation of terrestrial ecosystem responses to global change: mini-review. - Fungal Ecol. 10: 3-19.

Moore, D. et al. 2008. Fruit bodies: their production and development in relation to environment. - In: Boddy, L. et al. (eds), Ecology of saprotrophic basidiomycetes. Elsevier, pp. 79-103.

Norros, V. et al. 2014. Do small spores disperse further than large spores? - Ecology 95: 1612-1621.

Ohenoja, E. 1989. Effect of winter conditions on mushroom production. - Mem. Soc. Fauna Flora Fenn. 65: 77-80.

Ovaskainen, O. et al. 2016. Uncovering hidden spatial structure in species communities with spatially explicit joint species distribution models. - Methods Ecol. Evol. 7: 428-436.

Peter, M. et al. 2001. Nitrogen addition in a Norway spruce stand altered macromycete sporocarp production and below-ground ectomycorrhizal species composition. - New Phytol. 149: 311-325.

Pollock, L. J. et al. 2014. Understanding co-occurrence by modelling species simultaneously with a Joint Species Distribution Model (JSDM). - Methods Ecol. Evol. 5: 397-406.

Supplementary material (Appendix ECOG-03233 at < www. ecography.org/appendix/ecog-03233>). Appendix 1-4.
Primicia, I. et al. 2016. Linkages between climate, seasonal wood formation and mycorrhizal mushroom yields. - Agric. For. Meteorol. 228: 339-348.

Quine, C. et al. (eds) 2011. Woodlands. - NEP-WCMC.

Richardson, A. D. et al. 2010. Influence of spring and autumn phenological transitions on forest ecosystem productivity. - Phil. Trans. R. Soc. B 365: 3227-3246.

Rousk, J. and Bååth, E. 2011. Growth of saprotrophic fungi and bacteria in soil. - FEMS Microbiol. Ecol. 78: 17-30.

Rue, H. et al. 2009. Approximate Bayesian inference for latent Gaussian models by using integrated nested Laplace approximations. - J. R. Stat. Soc B 71: 319-392.

Schenk-Jäger, K. M. et al. 2016. Introducing mushroom fruiting patterns from the Swiss National Poisons Information Centre. - PLoS One 11: e0162314.

Stewart, J. R. 2009. The evolutionary consequence of the individualistic response to climate change. - J. Evol. Biol. 22: 2363-2375.

Taheri, S. et al. 2016. Did British breeding birds move north in the late 20th century? - Clim. Change Resp. 3: 5.

Tedersoo, L. et al. 2014. Global diversity and geography of soil fungi. - Science 346: 1256688.

ter Braak, C. J. F. et al. 2017. A critical issue in model-based inference for studying trait-based community assembly and a solution. - PeerJ 5: e2885.

Vellinga, E. C. et al. 2009. Global patterns of ectomycorrhizal introductions. - New Phytol. 181: 960-973.

Ward, D. F. 2014. Understanding sampling and taxonomic biases recorded by citizen scientists. - J. Insect Conserv. 18: 753-756.

Warton, D. I. et al. 2015. CATS regression - a model-based approach to studying trait-based community assembly. - Methods Ecol. Evol. 6: 389-398.

Wilson, R. J. et al. 2005. Changes to the elevational limits and extent of species ranges associated with climate change. - Ecol. Lett. 8: $1138-1146$.

Wolfe, B. E. and Pringle, A. 2012. Geographically structured host specificity is caused by the range expansions and host shifts of a symbiotic fungus. - ISME J. 6: 745-755.

Yan, Y. et al. 2017. Range shifts in response to climate change of Ophiocordyceps sinensis, a fungus endemic to the Tibetan Plateau. - Biol. Conserv. 206: 143-150. 